\title{
Analfabetismo científico funcional y la enseñanza universitaria de la Carrera de Derecho
}

\section{Functional scientific illiteracy and the university education of the Law Career}

\author{
WUILBER JORge AlCA ROBles \\ walcar@unmsm.edu.pe \\ https://orcid.org/0000-0001-6565-5391 \\ Universidad Nacional Mayor de San Marcos - Perú
}

\section{RESUMEN:}

En el contexto actual de los avances de la ciencia y la tecnología se visibiliza la existencia de un mayor número de personas analfabetas científicos y su agravante el nocivo crecimiento de los denominados analfabetos científicos funcionales, estudiando su incidencia y efectos en la enseñanza universitaria de los estudiantes de la carrera profesional del derecho. En el presente trabajo de investigación se aplica el enfoque aplicado bajo el método hermenéutico, con un estudio de fuentes y recopilación, organización, selección, lectura y análisis de material de carácter bibliográfico tanto nacional como extranjero, con la finalidad de realizar su interpretación en gabinete, contrastando la información obtenida con la indagación de las fuentes bibliográficas consultadas y la actual educación universitaria.

\section{ABSTRACT:}

It is analyzed in the context of current advances in science and technology, the existence of a greater number of illiterate scientists and its aggravating factor, the harmful growth of so-called functional scientific illiterates, studying its incidence and effects on university teaching of the students of the professional career of law. In this research work, the approach applied under the hermeneutic method is applied, with a study of sources and a compilation, organization, selection, reading and analysis of national and foreign bibliographic material, in order to perform its interpretation in the office, contrasting the information obtained with the investigation of the bibliographic sources consulted and the current university education.

\section{Palabras Clave:}

Analfabetismo científico; analfabeta científico funcional; enseñanza del derecho.

\section{KEYWORDS:}

Scientific illiteracy; functional scientist illiterate; law teaching. 


\section{Introducción}

En un mundo moderno, la ciencia, la tecnología y el flujo de información dominan el tiempo en casi todas las actividades y labores que realizan las personas en su vida diaria; por lo que sería impensable, no entender que, existe una directa relación entre el hombre y la ciencia o al menos en los beneficios que esta le prodiga en su quehacer diario y cotidiano.

No obstante, considerando esta vorágine de desarrollo e información, es fácil afirmar que "en nuestros días somos demasiado numerosos como para poder sobrevivir en las naciones modernas sin energía, abrigo, alimentos, medicina y tecnología, productos derivados de la ciencia" (Martínez, 2016, párr. 4). Muchos entendidos en el tema refieren que persiste el oscurantismo científico en la población en general, porque "pese al progreso logrado, en esta sociedad autodenominada tecnológica y de la información persisten actitudes irracionales (pseudociencia), y la incultura científica, al contrario del analfabetismo normal, todavía no ha sido desterrada" (Cabral, 2001, párr. 1).

Por lo anteriormente expuesto, sería insuficiente el uso irracional y casi de forma mecánica de los bienes, herramientas y servicios que la ciencia nos proporciona. El uso negligente de la ciencia por algunas personas, los puede llevar a un total desconocimiento a las reglas más elementales y conocimientos más básicos de su existencia e incluso ante la necesidad de su empleo o de su propia creación. Dichos escenarios pueden sumir en diversas situaciones a las sociedades, como: crisis económicas mundiales, sobrepoblación, ausencia de recursos renovables, la emergencia ambiental, las injusticias sociales, entre otros.

Si en nuestra población, el panorama es lúgubre, en general, pensemos por un momento, qué está ocurriendo con nuestros estudiantes universitarios, egresados y profesionales formados en las distintas carreras universitarias; en donde los avances de la ciencia y la tecnología son parte importante - al menos en lo formal - de su enseñanza y formación como futuros profesionales competentes. No obstante, la realidad de muchos de ellos, es que podrán ser analfabetos científicos; es decir, personas que usan los medios y bienes que la ciencia nos brinda, sin siquiera saber las nociones más elementales de su funcionamiento o lo que es peor, sin cuestionarla bajo los fundamentos que ella misma proporciona.

Para quienes ejercemos la docencia universitaria, es fácil evidenciar en dicho escenario, las actuales carencias de nuestros estudiantes que penosamente advertimos en las diferentes etapas de su formación. Ellos, en muchos casos, poseen serias dificultades para redactar y argumentar; desconociendo las más elementales reglas gramaticales, como: la sintaxis, el circunloquio, paráfrasis, los signos de puntuación, la ortografía, el manejo de términos propios de su carrera, entre otros. Dicha situación se agrava, si además de ello, muchos de estos alumnos se muestran como analfabetos científicos funcionales, que es un tipo muy distinto de analfabetismo científico y quizás más nocivo que este mismo.

Precisamos que ellos, a pesar de haber recibido una formación científica formal, podrían precarizar su situación al no comprender algunos conceptos, como, por ejemplo: qué es la ciencia, su fundamento o métodos, el planteamiento de un problema, hipótesis de trabajo, identificación de variables, entre otros. Entonces, si ya existe una grave limitación formativa en estos estudiantes, se vuelve más complicado esperar que ellos se desenvuelvan de mejor manera ante el conocimiento de la ciencia, sus fundamentos y métodos.

Por otro lado, existe un sostenido crecimiento de estudiantes y profesionales a nivel nacional, aunque se ha podido advertir una crisis lingüística real y de conocimiento científico, que se puede evidenciar en la formación universitaria. Dicha situación, que consideramos se viene agravando profundamente, es de conocimiento general, tanto en los fueros académicos como en el ejercicio profesional. Por ello, se han manifestado diversas voces alertando sobre esa crisis en diferentes niveles en muchos estudiantes de derecho, egresados y abogados de nuestro país.

La situación anterior se puede apreciar en la formación lingüística y científica de algunas personas, al no poder afrontar, analizar y resolver un problema de naturaleza jurídica, bajo bases científicas, lingüísticas y 
comunicativas adecuadas e idóneas. Estas se observan en la identificación del problema, de las herramientas jurídicas a utilizar para su solución, qué variables e indicadores en el ámbito del derecho se pueden aplicar, el planteamiento de hipótesis jurídicas, sin caer en falacias, la redacción de enunciados y argumentos claros además de concisos, bajo una correcta sintaxis y paráfrasis, entre otros. Ellas terminan por evidenciarse negativamente en el ejercicio profesional de jueces, fiscales, funcionarios, asesores, abogados en ejercicio y demás profesionales con esta especialidad, tal como lo refieren algunos juristas, estudiosos de estos temas.

Adicionalmente, ello se visualiza en el análisis de algunas demandas judiciales, efectuados por letrados colegiados y especializados en materia procesal, donde se verifica a detalle, la existencia de un inadecuado uso de la ortografía, abundantes circunloquios, pleonasmos y un uso inadecuado de las mayúsculas, géneros y números; además, de tener un contenido poco claro e impreciso. Sobre ello, Misari (2019) refiere la existencia de escritos del Ministerio Público, efectuados por algunos fiscales, abogados especializados en materia penal y procesal nombrados por el Estado, los cuales evidencian deficiencias en su construcción y redacción. Siendo esto último visible, en la redacción de textos que en un solo párrafo poseen varios argumentos o narraciones, que pueden separarse en párrafos independientes más breves y bajo un lenguaje más sencillo, pero en muchos casos abusan del tecnicismo y latinismo (Bullard y Mac Lean, 2002).

En ese mismo sentido, existe la emisión de resoluciones judiciales, con calidad de sentencia que son emitidos por algunos jueces y abogados nombrados por el Estado para ejercer dicha función tan trascendental para nuestra sociedad. Además, estos magistrados hacen gala de sus deficiencias argumentativas y su evidente ausencia en competencias gramaticales. El caso más representativo, es la sentencia emitida por el Juez Malzon Urbina (Municipalidad Metropolitana de Lima contra los ex comerciantes mayoristas La Parada) donde se ha llegado a escribir que

si bien la prensa ha criticado y resaltado pasajes anecdóticos de este fallo - como las referencias al Quijote, Sancho Panza, Galileo Galilei o González Prada (a quien llama "Vargas Prada")-, uno de los aspectos graves de ésta es su inconsistencia argumentativa, pésima redacción y ortografía, es extremadamente difícil "descubrir" qué ha querido decir el juez con tal o cual argumento. (Lovatón, 2013, párr. 8)

En consecuencia, queda establecida la relevancia de estudio del presente estudio teórico y la importancia de aportar alguna posición al respecto en materia de la enseñanza universitaria.

\section{Analfabetismo Científico Funcional}

En general, se debe entender que, a nivel educativo, se concibe al analfabeto como aquel individuo que no sabe leer ni escribir; es decir, que ignora y limita aspectos relativos a su propia la lengua materna y cultura. Por otro lado, existe el analfabetismo funcional, que se puede verificar en aquellos individuos que sabiendo leer y escribir, en la práctica no solo no leen, sino tienen limitaciones en la comprensión de lo leído, en su nivel más básico.

En la sociedad actual, dicha situación se encuentra presente en la realidad de los países en vías de desarrollo, siendo posible afirmar, con cierto grado de veracidad, que buena parte de la población también es analfabeta científica. Asimismo, dicha población vive de la ciencia y la tecnología, pero no la comprende, ni la percibe, ni mucho menos la evalúa, solo la consume. En esa lógica, Martínez (2016) refiere que en "nuestros días somos demasiado numerosos como para poder sobrevivir en las naciones modernas, con ausencias de energía, abrigo, alimentos, medicina y tecnología, productos derivados de la ciencia" (párr.4). De esta manera, estaríamos volviéndonos dependientes de ella, pero no sin entenderla, al menos en sus reglas y conocimientos más básicos.

El analfabetismo científico funcional, es aquel estado del individuo donde "predominan las destrezas, actitudes y el pensamiento en cuanto a la realidad material del conocimiento del día a día, teniendo el 
individuo poca capacidad para usar dichos conocimientos referentes a la ciencia para entender los problemas" (Huete, 2017, p.12). Este tipo de analfabetismo también se puede definir como aquellas falencias en la formación científica que se muestran en los estudiantes y graduados universitarios, que muestran incapacidad de producir no solo conocimiento, sino también poder evaluarlo o aplicarlo (García, 2016). De la misma forma, también sería entenderla como aquellas destrezas y actitudes de un sujeto sobre la realidad material propios del conocimiento cotidiano, pero con escasa o nula capacidad de emplear sus escasos conocimientos relativos a las ciencias, para comprender los fenómenos y problemáticas de su entorno y menos actuar bajo una orientación científica (Molano, 2015).

Esa limitación se agrava en el ámbito formativo universitario donde este tipo de analfabetismo se evidencia en aspectos propios de su formación y producción académica. Al respecto García (2016) refiere que "esas falencias se manifiestan en tres aspectos: ausencia de una actitud o mente científica, dificultad para construir y manejar conceptos (deficiencia epistémica), y dificultad en la construcción de textos científicos (deficiencia discursiva)" (p. 60).

Algunos autores en vez de estudiar al analfabeto científico funcional, centran su interés y se enfocan en caracterizar y desarrollar su sentido positivo bajo el denominado alfabeto científico funcional. Este es definido, como aquel sujeto que evidencia nociones, entiende aspectos de la ciencia y la tecnología, que lo demuestra al tener familiaridad con algo del lenguaje utilizado en la ciencia sin pasar apuros, ni caer en fatales contradicciones. Así, Shamos (citado por Cabral, 2001), define tres tipos de alfabetas científicos, los cuales son: el alfabeta científico cultural (posee un conocimiento pasivo de los términos científicos y se le hace familiar algunos términos empleados en esta); alfabeta científico funcional (usa conceptos de la ciencia y aplica los mismos en la comprensión de lectura con contenido científico), y el alfabeta científico verdadero (comprende términos de la ciencia, hace cálculos y denota competencias científicas).

Al margen de las discusiones terminológicas, lo cierto es que, la problemática es más crítica a este nivel, porque en general, es grave en nuestra población. Pensemos un poco, qué destino nos depara como sociedad moderna, si la propia reserva profesional en nuestros países, el segmento de estudiantes universitarios y futuros profesionales, terminan por ser clasificados en muchos casos como unos analfabetos científicos funcionales. En otro sentido, es mencionar que los estudiantes o profesionales, quienes a pesar de haber recibido en las aulas universitarias y en diferentes etapas de su formación una enseñanza científica formal (sea buena o mala y de lo cual se podría hacer un estudio posterior), podrían tener dificultades para comprender cabalmente lo que es la ciencia, ni el fundamento de sus métodos, agravando con ello la propia condición del analfabetismo científico en general.

De la misma forma, este tipo de analfabetismo podría repetirse en los niveles de postgrado, estudio cuya tarea será de seguro más intensa y complicada. Y en lo que adicionalmente, algunos autores refieren que atender una alfabetización científica en los niveles superiores de posgrado se volvería una tarea compleja y distante, de seguro muy complicada (Vázquez, Acevedo y Manassero, 2005).

Por tanto, el objetivo debe ser eliminar el analfabetismo científico funcional en nuestras aulas universitarias y con ello, mejorar la divulgación del conocimiento científico, para que estos estudiantes y futuros profesionales, sepan qué es la ciencia. Además de conocer, cuál es su estructura, cómo funciona y particularmente, cómo se aplica en la mejora de la vida en sociedad, más como un medio necesario y motor de cambio. A decir de algunos "un elemento esencial para que los egresados de cualquier institución se desempeñen en su labor profesional y sean competitivos a nivel internacional es, precisamente, el que cuenten con una cultura científica mínima para poder desenvolverse en el siglo XXI" (Cabral, 2001, p.13).

Siguiendo a Molano (2015) podemos aseverar una real alfabetización tecno científica de la ciudadanía, bajo una verdadera cultura científica que implicaría el desarrollo de competencias para la participación de todos los ciudadanos; ello mejoraría la toma de decisiones relacionadas con el desarrollo tecno científico. Es aquí, donde los científicos e investigadores con enfoque social, deben liderar esa participación activa sobre lo 
que se espera, se desea y se necesita de la ciencia y la tecnología en nuestra realidad actual. De esta manera, se estaría ayudando a la población en general, en las decisiones respecto a la vida, la salud, la educación, el medio ambiente, la igualdad, entre otros.

Tener más alfabetos científicos funcionales, nos permitiría, tener una reserva de potenciales investigadores que hagan ciencia, pero con ética social, conscientes de su rol en la sociedad. Ello, bajo el paradigma irrefutable de que la alfabetización científica es un derecho de todas las personas y un requisito para considerarse ciudadano responsable de la sociedad, logrando tener además una nueva generación de científicos investigadores, superando la idea equivocada que asimila como sinónimos los términos de científico e investigador, esto sería lo ideal (Cereiijido, 2009). Esto no debe ser así, debido a que no es acertado asumir a un científico que no tenga creatividad e innovación o a un investigador que no tenga una visión científica del mundo, considerar ello sería fatal en nuestra realidad.

\section{El Analfabetismo Científico Funcional en la Enseñanza del Estudiante de Derecho: Hacia una Nueva Pedagogía}

Como bien sabemos, la carrera profesional de Derecho y la abogacía está íntimamente vinculadas a la historia de la propia humanidad. El Derecho responde por su propia definición, a aquella ciencia social que regula las relaciones humanas de convivencia social y permite resolver los conflictos de intereses y la incertidumbre jurídica, bajo un orden normativo e institucional de la conducta humana en sociedad, y siempre inspirado en postulados de justicia, equidad e igualdad entre otros valores. Se la puede definir entonces como el

conjunto de normas jurídicas positivas que surge de la sociedad como un producto cultural generado dentro de leyes y que tienen la finalidad de regular las relaciones entre los miembros de esa sociedad - las personas - y de estas con el Estado (Orna, 2011, p.2).

En consecuencia, es innegable afirmar que, el derecho es una ciencia y como tal se encuentra provista de teorías, métodos, principios y procedimientos que se desarrollan sobre las conductas humanas en sociedad, regulando normas que conjugan el hecho, valor y norma. Es necesario mencionar que, no es una ciencia similar a las ciencias formales, debido a que no podríamos afirmar que su objeto es único e inmutable. Se hace necesario tener en cuenta que la ciencia del derecho regula conductas humanas con contenido jurídico, y en ese sentido, siendo una ciencia, debe regular bajo un necesario rigor científico estas situaciones y relaciones jurídicas cambiantes y mutables. Cobra sentido, lo afirmado por los profesores Bullard y Mac Lean (2002) cuando se refieren que el objeto y naturaleza de la ciencia del derecho "es consecuencia de la creación del hombre. El Derecho es creado por el hombre y su "naturaleza" y la de las instituciones llega a cambiar tan pronto el legislador cambia la Ley que la regula" (p.3).

A pesar que vivimos en el siglo de los cambios constantes, de la información en tiempo real, del mundo globalizado, de los avances científicos de última generación, es penoso que todavía en muchas universidades se siga enseñando el derecho bajo una óptica de contenidos, más que de competencias; además, priorizando la memoria sobre el análisis. Y donde el dictado de clases es más un reproducir, trasladar información y contenidos, que el motivar y estimular el análisis y la crítica informada, un inconcebible culto a la memoria reflejada no solo en su propia enseńanza, sino también en las formas de evaluación y la manera de calificar el resultado.

De todo lo ya expuesto, es evidente pensar que estamos como estamos, justamente porque no le hemos prestado la atención debida a esta problemática en la formación universitaria de nuestros alumnos en general y más aún en nuestros estudiantes de la carrera profesional de Derecho.

Las razones y fundamentos de esta problemática ya han sido expuestos anteriormente y su incidencia negativa en la actual enseñanza y formación de los estudiantes de Derecho. Siendo evidente y de larga data 
que a la fecha tengamos no solo estudiantes y egresados, sino varias generaciones de profesionales abogados, que presentan deficiencias en su formación lingüística y científica. Esta situación se visibiliza en que no pueden afrontar, analizar y resolver un problema de naturaleza jurídica bajo bases científicas, lingüísticas y comunicativas adecuadas e idóneas, que no saben cómo identificar un problema con relevancia jurídica o qué herramientas jurídicas utilizar para su solución. Además, el conocimiento de qué variables e indicadores en el ámbito del derecho aplicar, plantear hipótesis jurídicas, redactar enunciados y argumentos claros y concisos y demás competencias y habilidades necesarias para un adecuado e idóneo ejercicio de esta noble profesión.

Entonces, nos surge la siguiente duda ¿̇cómo incorporar este nuevo modelo en el diseño curricular de la enseñanza del Derecho? ¿cómo internalizar este nuevo paradigma en el pensamiento de las autoridades, los docentes y alumnos actores en el proceso de formación del profesional de Derecho? Para ello, consideramos que en pleno siglo XXI, debemos reinventarnos y esto inicia por cambiar el modelo de enseñanza del Derecho que alberga formas tradicionales, como el dictado únicamente de clases magistrales, los dictados solo de contenidos, en aulas ordenadas de forma militar con carpetas unas detrás de otra, frente una pizarra acrílica o considerando que lo último de la tecnología, es el usar el proyector multimedia o videos del curso. Si bien los contenidos y la información son importantes; pero estos en solitario, ya no responden a la forma moderna de una verdadera enseñanza del Derecho.

Se hace necesario implementar una nueva pedagogía, reestructurar todo el plan de estudios en la enseńanza del Derecho, creando unidades de aprendizaje y de competencia, que a su vez tengan objetivos generales y específicos, pero basados en el logro de competencias, habilidades y destrezas legales, más que de solo contenidos. Asimismo, desarrollar con más énfasis los cursos interdisciplinarios, así como la implementación del método de razonamiento inductivo, llamado de solución de casos, identificando para ello el interés del alumno y concordándolo con la motivación ontológica de la esencia de nuestra profesión.

De la misma forma, la necesidad de precisar un nuevo enfoque basado en la evaluación formativa, para promover el aprendizaje a través de la experiencia, los logros del aprendizaje, el análisis de las competencias y un nuevo diseño de la enseñanza (Mac Lean, 2012). Se hace necesario que los docentes puedan asumir un rol de liderazgo, plantear en el aula: retos y metas logrables en la aplicación práctica de las ciencias jurídicas, siendo conscientes de la realidad del mercado laboral donde estos futuros profesionales puedan laborar.

En consecuencia, lograr alfabetas científicos funcionales en Derecho o los llamados alfabetas científicos verdaderos en términos de Shamos (citado por Cabral, 2001), no es una tarea fácil. No es buscar formar profesionales con la única intención que sean científicos o investigadores, no es nada de eso, sino es formar estudiantes que asuman el compromiso de ser futuros profesionales de derecho, con formación y vocación de científicos e investigadores, que puedan integrar los contenidos de la teoría y la práctica jurídica sin mayor dificultad. Todo ello, sin alejarse del rigor científico y fortaleciendo con ello, su libertad de pensamiento, sana, crítica y en independencia con valores éticos y espíritu democrático.

De llegar a la situación anteriormente mencionada, tendríamos una reserva de profesionales alfabetas científicos funcionales en Derecho que, al momento de ejercitar su profesión, demuestren a la sociedad que tiene una idónea formación del Derecho, pero con un alto nivel ético, un profesional con sólidas bases en el quehacer científico e investigación. Asimismo, que engrandezca nuestra labor como profesionales, devolviéndonos el respeto y reconocimiento social que se le debe a nuestra condición de abogados.

En esa misma lógica es necesario precisar que el término abogado proviene del latín advocatus que significa ayuda, y que propiamente significa "aquel que advoca, defiende, intercede, habla en favor de alguien" (Terragni, 2018, párr. 2). Con ello, es posible afirmar que un estudiante, futuro abogado, sería un defensor de intereses, un jurista formado para la defensa y protección de las personas o del propio Estado en sus diversos niveles. De esta manera, se revertería el pésimo concepto que se le tiene hoy, por la sociedad en general, asociando a los abogados, profesionales del Derecho en temas de corrupción, desprestigio, crisis del sistema de justicia, inadecuadas prácticas legales. 
Esa percepción del mundo del Derecho, es aquella que refiere que Del Mastro (2018) cuando refiere que,

en nuestro país, el mundo del derecho está cada vez más desprestigiado, lo mismo que la profesión del abogado, la política y, en general, todo lo que se refiere al Estado y las leyes. El nivel ético de los profesionales del derecho es preocupante y contribuye a este desprestigio. (p. 466)

Cumplir la ley, es un deber de todo ciudadano, y más aun de los estudiantes de Derecho futuros abogados. El sentido de responsabilidad es igual también para todos los que queremos un sistema universitario y de enseñanza del Derecho que contribuya al desarrollo del país. Precisamos que cumplir la ley también es un ejercicio ciudadano y más si lo realiza un profesional del Derecho con sólida formación jurídica y científica, de seguro lograr eso nos debe hacer una mejor sociedad, en lo jurídico y legal.

\section{Método}

El presente artículo es carácter teórico orientado a la recolección de información de tipo descriptivo explicativo en el marco de una investigación eminentemente documental. El enfoque de investigación es cualitativo y se emplea el método hermenéutico pues se inicia con un estudio de fuentes y recopilación de material de carácter bibliográfico tanto nacional como extranjero, su organización, selección, lectura y análisis, para luego proceder a realizar su interpretación en gabinete. Luego, se contrasta la información obtenida con la indagación de las fuentes bibliográficas consultadas y la realidad educativa universitaria actual, siguiendo los procedimientos lógicos como son el análisis, síntesis, deducción e inducción sobre el tema (Palella y Martins, 2012, p.87).

\section{Discusión y conclusiones}

a. El analfabetismo científico funcional es aquel estado en que se encuentra un individuo donde evidencia poca capacidad para usar los conocimientos relativos a la ciencia, para entender los problemas de la realidad o que muestran incapacidad de producir no solo conocimiento, sino también de poder avaluarlo o aplicarlo.

b. En la actualidad, no solo estudiantes y egresados, sino varias generaciones de profesionales abogados, presentan deficiencias en su formación lingüística y científica, que les impide afrontar, analizar y resolver un problema de naturaleza jurídica bajo bases científicas, lingüísticas y comunicativas adecuadas e idóneas. Ello, trae consigo que no se pueda identificar fácilmente un problema con relevancia jurídica o qué herramientas jurídicas utilizar para su solución, qué variables e indicadores en el ámbito del derecho aplicar, plantear hipótesis jurídicas, redactar enunciados y argumentos claros y concisos y demás competencias, así como habilidades necesarias para un adecuado e idóneo ejercicio de esta noble profesión.

c. Lograr alfabetas científicos funcionales en Derecho, no es buscar formar profesionales con la única intención que sean científicos o investigadores, es formar estudiantes que asuman el compromiso de ser futuros profesionales del Derecho. Todo ello, con formación y vocación de científicos e investigadores, que puedan integrar los contenidos de la teoría y la práctica jurídica sin mayor dificultad y sin alejarse del rigor científico, pero fortaleciendo con ello, su libertad de pensamiento, sana crítica, valores éticos y espíritu en democracia.

d. Se debe implementar una nueva pedagogía, reestructurar todo el plan de estudios en la enseñanza del Derecho, creando unidades de aprendizaje y de competencia, que a su vez tengan objetivos generales y específicos; pero basados en el logro de competencias, habilidades y destrezas legales y no solamente contenidos. Este contexto permite el desarrollo con más énfasis de los cursos interdisciplinarios, implementando el método de razonamiento inductivo, denominado de solución de casos. 
e. La responsabilidad es compartida, tanto por los estudiantes de Derecho, futuros abogados, como por aquellos que queremos un sistema universitario y de enseñanza del Derecho que contribuya al desarrollo del país, debido a que cumplir la ley si bien es un ejercicio ciudadano, es más significativo si lo realiza un profesional del Derecho con una sólida formación jurídica y científica.

\section{Referencias}

Bullard, A., y Mac Lean, A. (2002). La enseñanza del Derecho: ¿Cofradía o archicofradía? Academia. Revista sobre enseñanza del Derecho, (13),21-47 https://dialnet.unirioja.es/servlet/articulo?codigo=3743478

Cabral, I. (2001). Alfabetismo científico y educación. Revista Iberoamericana de Educación. https://rieoei.org/historico/ deloslectores/Cabral.PDF

Cereijido, M. (2009). La Ciencia como Calamidad. Buenos Aires, Argentina: Gedisa.

Del Mastro, F. (2018). Venga a nosotros tu reino: la justicia como fuerza anímica ausente en la enseñanza del derecho. Revista de Derecho de la PUCP, (81), 463-510. doi: 10.18800/derechopucp.201802.015

García, B. (2016). Enfoque Epistémico y Discursivo en el Currículum Universitario. Revista Científica Hallazgos 21, 2(1), 58-65. https://revistas.pucese.edu.ec/hallazgos21/

Huete, M. (2017). Análisis sobre el tratamiento de la alfabetización científica desde edades tempranas en revistas especializadas. (Trabajo de Fin de grado), Universidad de Valladolid. http://uvadoc.uva.es/handle/10324/29760

Lovatón, D. (2013). Emboscada Judicial en la Parada. Recuperado de https://www.alainet.org/es/active/64493

Mac Lean, A. (2012). Reforma de la Educación Legal en el Perú. Cuadernos de investigación, (17). http:// revistas.upc. edu.pe/index.php/sinergia/article/view/29

Martínez, M. (2016). Analfabetismo científico y sus consecuencias/La ciencia desde el Macuiltepetl. https:// plumaslibres.com.mx/2016/02/04/analfabetismo-cientifico-y-sus-consecuenciasla-ciencia-desde-elmacuiltepetl/\#:-:text=Analfabetismo\%20cient\%C3\%ADfico\%20y\%20sus\%20consecuencias $\% 2 \mathrm{FLa} \% 20$ ciencia\%20desde\%20el\%20Macuilt\%C3\%A8petl,-Por\%20Manuel\%20Mart\%C3\%ADnez\&text=La\%20 situaci $\% \mathrm{C} 3 \% \mathrm{~B} 3 \mathrm{n} \% 20 \mathrm{deb} \% \mathrm{C} 3 \% \mathrm{ADa} \% 20$ alarmarnos\%20pues,elemento\%20indispensable $\% 20 \mathrm{de} \% 20 \mathrm{la} \% 20$ supervivencia

Misari, D. (2019). Errores Gramaticales en la Demanda de Sheyla Rojas.https://dmisari.blogspot.com/2019/04/erroresgramaticales-en-la-demanda-de.html

Molano, A. (2015). El fomento de la alfabetización científica mediante el trabajo con estudiantes de secundaria Secuencia de actividades para el desarrollo de una problemática ambiental en Química Universidad Pedagógica Nacional, Maestría en Docencia de la Química. http://repository.pedagogica.edu.co/handle/20.500.12209/266

Orna, O. (2011). ¿Quées el derecho? ¿El Derecho es una ciencia?http://revistas.urp.edu.pe/index.php/Inkarri/article/view/538

Palella, S., y Martins, F. (2012). Metodología de la Investigación Cuantitativa (3ra. Ed.). Caracas, Venezuela: Fondo Editorial de la Universidad Pedagógica Experimental Libertador.

Terragni, M. (2018). Advocatus, Lawyer, Rechtsanwalt. https://www.terragnijurista.com.ar/libros/advocatus.htm

Vázquez, Á., Acevedo, J., y Manassero, M. (2005). Más allá de la enseñanza de las ciencias para científicos: hacia una educación científica humanística. Revista Electrónica de Enseñanza de las Ciencias, 4(2).https://www. researchgate.net/publication/28087406_Mas_alla_de_la_ensenanza_de_las_ciencias_para_cientificos_hacia_ una_educacion_cientifica_humanistica

\section{Como citar este artículo:}

Alca, W. (2021). Analfabetismo científico funcional y la enseńanza universitaria de la Carrera de Derecho. Revista Peruana de Investigación e Innovación Educativa, 1 (1), 44-60 